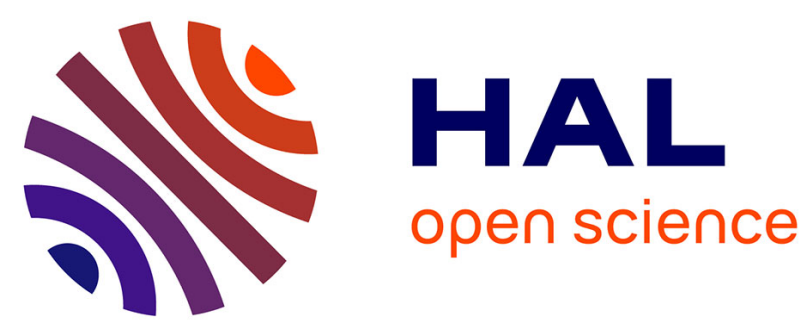

\title{
Post-pinch-off relaxation of two-dimensional droplets in a Hele-Shaw cell
}

\author{
Dhirendra Tiwari, Lionel Mercury, Marcel Dijkstra, Himanshu Chaudhary, \\ José-Federico Hernandez-Sanchez
}

\section{To cite this version:}

Dhirendra Tiwari, Lionel Mercury, Marcel Dijkstra, Himanshu Chaudhary, José-Federico HernandezSanchez. Post-pinch-off relaxation of two-dimensional droplets in a Hele-Shaw cell. Physical Review Fluids, 2018, 3 (12), Article: 124202 (12 p.). 10.1103/physrevfluids.3.124202 . insu-01950861

\section{HAL Id: insu-01950861 https://hal-insu.archives-ouvertes.fr/insu-01950861}

Submitted on 19 Dec 2018

HAL is a multi-disciplinary open access archive for the deposit and dissemination of scientific research documents, whether they are published or not. The documents may come from teaching and research institutions in France or abroad, or from public or private research centers.
L'archive ouverte pluridisciplinaire HAL, est destinée au dépôt et à la diffusion de documents scientifiques de niveau recherche, publiés ou non, émanant des établissements d'enseignement et de recherche français ou étrangers, des laboratoires publics ou privés. 


\title{
Post-pinch-off relaxation of two-dimensional droplets in a Hele-Shaw cell
}

\author{
Dhirendra Tiwari, ${ }^{1,2,3, *}$ Lionel Mercury, ${ }^{1}$ Marcel Dijkstra, ${ }^{4}$ Himanshu Chaudhary, ${ }^{5}$ \\ and José Federico Hernández-Sánchez ${ }^{6,7}$ \\ ${ }^{1}$ Institut des sciences de la Terre d'Orléans, UMR 7327 Université d'Orléans, CNRS, BRGM, 1A rue de la \\ Férollerie, 45071 Orléans, France \\ ${ }^{2}$ Mesoscale Chemical Systems Group, MESA + Institute for Nanotechnology, University of Twente, \\ P.O. Box 217, 7500 AE Enschede, The Netherlands \\ ${ }^{3}$ The BIOS Lab-on-a-Chip Group, MESA+ Institute for Nanotechnology, University of Twente, \\ P.O. Box 217, 7500 AE Enschede, The Netherlands \\ ${ }^{4}$ Faculty of Electrical Engineering, Mathematics and Computer Science (EEMCS), University of Twente, \\ P.O. Box 217, 7500 AE Enschede, The Netherlands \\ ${ }^{5}$ School of Engineering Sciences in Chemistry, Biotechnology and Health, Dept. Chemistry, Division of \\ Applied Physical Chemistry, KTH Royal Institute of Technology, SE-10044 Stockholm, Sweden \\ ${ }^{6}$ Instituto de Ciencias Aplicadas y Tecnología, Universidad Nacional Autónoma de México, Circuito Exterior \\ S/N, Ciudad Universitaria, A.P. 70-186, Delegación Coyoacán, C.P. 04510, Ciudad de México, México \\ ${ }^{7}$ Physics of Fluids group, MESA+Institute for Nanotechnology, J. M. Burgers Centre for Fluid Dynamics, \\ University of Twente, P.O. Box 217, 7500 AE Enschede, The Netherlands
}

(Received 8 January 2018; published 18 December 2018)

\begin{abstract}
We report on the shape relaxation of two-dimensional (2D) droplets, formed right after the spontaneous pinch-off of a capillary bridge droplet confined within a Hele-Shaw cell. An array of bridge droplets confined within a microchip device first undergoes neck thinning due to the evaporation-driven volume change. Subsequently, an abrupt topological change transforms each bridge droplet into a small central satellite droplet and the twin droplets pinned at the edges of the cell. We monitor the shape relaxation with high-temporal-resolution optical microscopy. Capillary action drives the 2D shape relaxation, while the viscous dissipation in the film retards it. As a result, the tip of the twin droplets exhibits a self-similar parabolic shape evolution. Based on these observations, the lubrication-approximation model accurately predicts the internal pressure evolution and the droplet tip displacement. The geometrical confinement substantially slows down the dynamics, facilitating visualization of the capillary-viscous regime, even for low-viscosity liquids. The characteristic relaxation timescale shows an explicit dependence on the confinement ratio (width/gap) and the capillary velocity of liquid. We verify the broad applicability of the model using different liquids.
\end{abstract}

DOI: 10.1103/PhysRevFluids.3.124202

\section{INTRODUCTION}

In 1840, Plateau conducted a series of seminal experiments, immersing macroscopic oil droplets into a mixture of water and alcohol of the same density [1]. These experiments not only established the molecular origin of capillarity but also showed that the mean curvature multiplied by the surface tension acts as the restoring force [1,2]. The dynamics of liquid interface is a problem of general interest and has been studied in a wide variety of contexts, including coating films [3], fragmentation

\footnotetext{
*Corresponding author: dhirendra.tiwari@cnrs-orleans.fr
} 
[4], atomization [5], foam stability [6], and even the fizz of champagne [7]. Additionally, the retraction of liquid film has appeared in a broad spectrum of physical phenomena, such as in fuel injectors [8], dynamics of droplets in laboratory-on-a-chip devices [9], foams used in the food industry, and even biological membranes [10].

The majority of liquid film retraction studies involve a freely suspended film, which retracts once a hole has nucleated on its surface, hereinafter referred to as the "Taylor-Culick" experiment [11,12]. The examples include bursting of a small [13-15] or giant soap bubble [16] and the thickening of two-dimensional (2D) polymer sheets [17,18]. The early Taylor-Culick experiments with soap films showed mass accumulation in a nearly cylindrical rim at the edge $[13,14,19]$. The rim, which is thicker, moves, while the liquid in the film remains stationary. Thus, the global momentum balance compares the rate of rim-momentum change and the capillary force per unit length along the edge, $\left(\rho H v_{T C}\right) v_{T C}=2 \sigma$, where $\rho$ is the density, $\sigma$ is the surface tension, $H$ is the film thickness, and $v_{T C}$ is the Taylor-Culick velocity [19]. More recent experiments with extremely viscous liquids [17,18] are explained by the numerical study of retracting twodimensional planar sheets $[20,21]$ for an arbitrary Ohnesorge number Oh, which compares the relative importance of capillary, viscous, and inertial forces. The Taylor-Culick experiment, from soap to extremely viscous films, involves an early capillary-viscous regime with a characteristic timescale $\tau_{T C}=\mu H / 2 \sigma$, where the viscous dissipation occurs near the edge, defined by a characteristic length scale $\mathrm{Oh} H$ or the Stokes length $\mu / \rho v_{T C}$. If the axial extent of the film is shorter than the Stokes length, the viscous effects extend to the entire film, causing the thickening without any rim formation. In nature, the surface tension of liquids remains within one order of magnitude [22], i.e., $10-100 \mathrm{mN} / \mathrm{m}$, but the viscosity $\mu$ may vary over many orders of magnitude. A free-standing $10-\mu \mathrm{m}$-thick soap film $\left(\mu=10^{-3} \mathrm{Pas}, \sigma=20 \mathrm{mN} / \mathrm{m}\right)$ and a polydimethylsiloxane (PDMS) film $\left(\mu=10^{+3} \mathrm{Pas}, \sigma=20 \mathrm{mN} / \mathrm{m}\right)$ yields a $\tau_{T C}$ on the order of $100 \mathrm{~ns}$ and $100 \mathrm{~ms}$, respectively. Thus, the early capillary-viscous regime has only been reported with extremely viscous liquids $[17,18,23,24]$.

Another set of film relaxation experiments study the dynamics of a stretched liquid puddle over a flat hydrophobic surface [2]. These films have a thickness on the order of millimeters (set by the capillary length) and the resulting dynamics is governed by a balance between capillary and inertial forces.

On a different platform, such as the laboratory-on-a-chip devices, droplets are generated, split, and merged depending on the application requirements [9]. Thus, understanding the relaxation dynamics of droplets in Hele-Shaw geometry is of technological importance [9]. The coalescence of droplets in Hele-Shaw geometry has been extensively studied, both numerically [25] as well as experimentally $[26,27]$. We report on the shape relaxation of $2 \mathrm{D}$ droplets formed right after the pinch-off of a bridge droplet confined in Hele-Shaw cell, driven by spontaneous evaporation. The gap of the cell is about one micron thick with slit geometry. The confinement leads to a major departure from the Taylor-Culick mechanism. First, a rim thicker than the film of liquid cannot fit within the gap between the walls of the Hele-Shaw cell. Second, this means that liquid has to flow over a long distance, from the moving tip in the center to the sides of the cell. Third, the driving pressure difference comes from the in-plane curvature of the interface, because the out-of-plane curvature is constant, being the gap thickness of the cell. Finally, velocity variations across the cell gap determine the rate of viscous dissipation. Based on these observations, we develop a phenomenological model to estimate the characteristic timescale, which shows an explicit dependence on the confinement ratio and the capillary velocity of the liquid. In Hele-Shaw geometry, the ratio of the width $W$ and the gap $H$ forms the confinement ratio $(C=W / H)$ while the ratio of surface tension and viscosity defines the capillary velocity $v_{C a}=\sigma / \mu$. Thus, we report on the observation of the capillary-viscous regime attributed purely to the effect of geometrical confinement, despite using low viscosity liquids. The confinement ratio dramatically delays the relaxation process, consistent with the characteristic 2D dynamics of the Hele-Shaw geometry. 


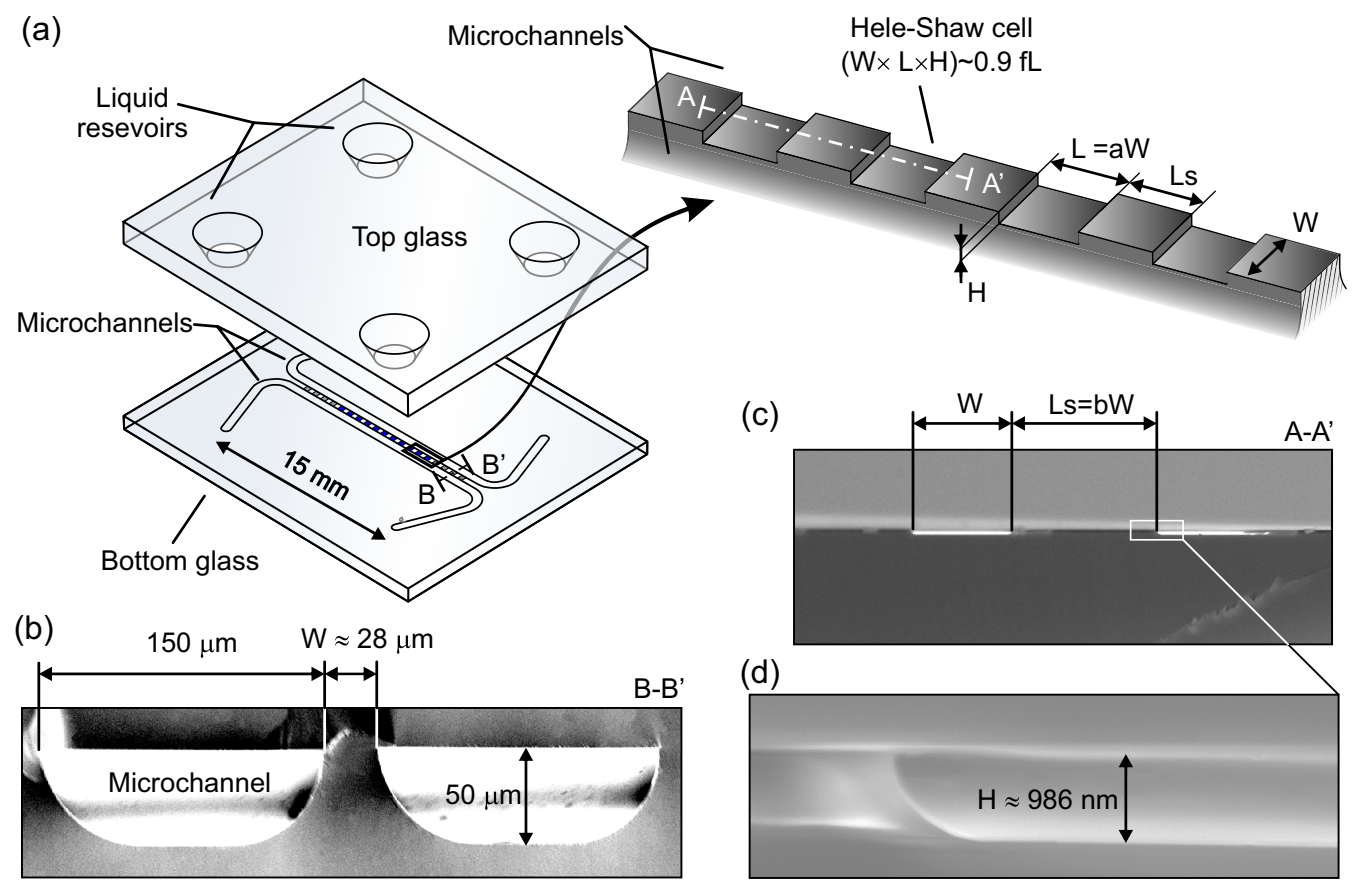

FIG. 1. (a) Schematic of the microchip. The top glass wafer contains reservoirs and the bottom glass wafer contains a set of parallel microchannels along with an array of bridging Hele-Shaw cells (top right inset). (b) SEM cross section of the U-shaped parallel microchannels (bottom left inset) as cut along B-B'. (c) The U-shaped bridging Hele-Shaw cell as cut along A-A'. (d) The inset of the Hele-Shaw cell edge.

\section{THE EXPERIMENT}

To study the relaxation of droplets in Hele-Shaw geometry, we utilize the microchip as depicted in Fig. 1 (see Fig. S1 in the Supplemental Material [28] for the optical image of the microchip). The microchip is fabricated from glass wafers using the etching technique, which imparts a dual-scale structure, corresponding to the nano- and microscales. It consists of a pair of parallel microchannels with the U-shaped cross section of $50 \mu \mathrm{m}$ deep, $150 \mu \mathrm{m}$ wide, and $1.5 \mathrm{~cm} \mathrm{long}$, as shown in Fig. 1(b). An array of bridging Hele-Shaw cells with height $H=986 \mathrm{~nm}$, width $W=28 \mu \mathrm{m}$, and length $L=32 \mu \mathrm{m}$ interconnect the two microchannels. The ratio of width $W$ and length $L$ is a fixed geometrical factor with $L / W \approx 1.14$. The separation between the nearest neighbor cell is 32 and $60 \mu \mathrm{m}$, corresponding to the two different microchip configurations [see Fig. 1(c)].

Prior to the experiment, the microchip is treated with air plasma to make it lyophilic, which ensures immediate liquid wicking. The capillary velocity $v_{C a}$ for water, ethanol, and isopropanol varies about one order of magnitude (see Table I). When exposed to the laboratory atmosphere (room temperature $298 \mathrm{~K}$ and constant relative humidity 50\%), the volatile liquid inside the microchip starts to evaporate.

The evaporation begins at the reservoirs (largest capillary radius), followed by the microchannels (intermediate microscale capillary radius) and finally in the array of Hele-Shaw cells [smallest capillary radius; see Figs. 2(a)-2(c)]. During the final stage of evaporation, each Hele-Shaw cell contains a 2D square droplet with a volume of about $L \times W \times H \approx 0.9 f \mathrm{~L}$ [see Fig. 1(c)]. The liquid in each cell remains interconnected with its neighboring cell via a pair of parallel, quasistatic, and perpetual nanoscopic corner films [see Figs. 2(a)III and 2(b)], as the Concus-Finn condition is satisfied for nearly perfect wetting liquids [29]. This condition states that the sum of the contact angle $(\theta \approx 0)$ and half the dihedral angle of the corner $(\alpha \approx \pi / 4)$ is less than $\pi / 2$, i.e., $\theta+\alpha<\pi / 2$. 
TABLE I. Liquid properties and depth-averaged Reynolds number.

\begin{tabular}{lccccc}
\hline \hline & $\begin{array}{c}\text { Surface } \\
\text { tension } \\
\sigma\end{array}$ & $\begin{array}{c}\text { Dynamic } \\
\text { viscosity } \\
\mu\end{array}$ & $\begin{array}{c}\text { Density } \\
{\left[\begin{array}{c}\mu \\
{[\mathrm{mPa}]}\end{array}\right.}\end{array}$ & $\begin{array}{c}\text { Capillary } \\
\text { velocity } \\
v_{C a} \\
{\left[\mathrm{Kg} / \mathrm{m}^{3}\right]}\end{array}$ & $\begin{array}{c}\text { Depth-averaged } \\
\text { Reynolds number } \\
\bar{R} \mathrm{e}\end{array}$ \\
\hline Istiquid & 21.0 & 2.04 & 10 & 786 & 0.000056 \\
Ethanol & 22.0 & 1.05 & 21 & 789 & 0.000210 \\
Water & 72.0 & 0.89 & 81 & 1000 & 0.001300 \\
\hline \hline
\end{tabular}

Further, a mechanical equilibrium at the junction of the corner film and the confined droplet within Hele-Shaw cell ensures Laplace pressure continuity. Corresponding to the pressure continuity requirement, the corner film radius orthogonal to the axial flow is roughly fixed by the Hele-Shaw gap size [see Fig. 2(b)]. The droplets not only undergo a direct evaporative loss to maintain the local vapor equilibrium but are also drained out by the nanoscopic corner films [30]. These corner films in turn feed the evaporative demand in the microchannel, as shown in Fig. 2(b). The net decrease in liquid volume transforms each square droplet into a bridge droplet, which undergoes neck thinning followed by a spontaneous pinch-off [see from top to bottom in the $(\mathrm{N}+1)$ th cell of Fig. 2(c)].

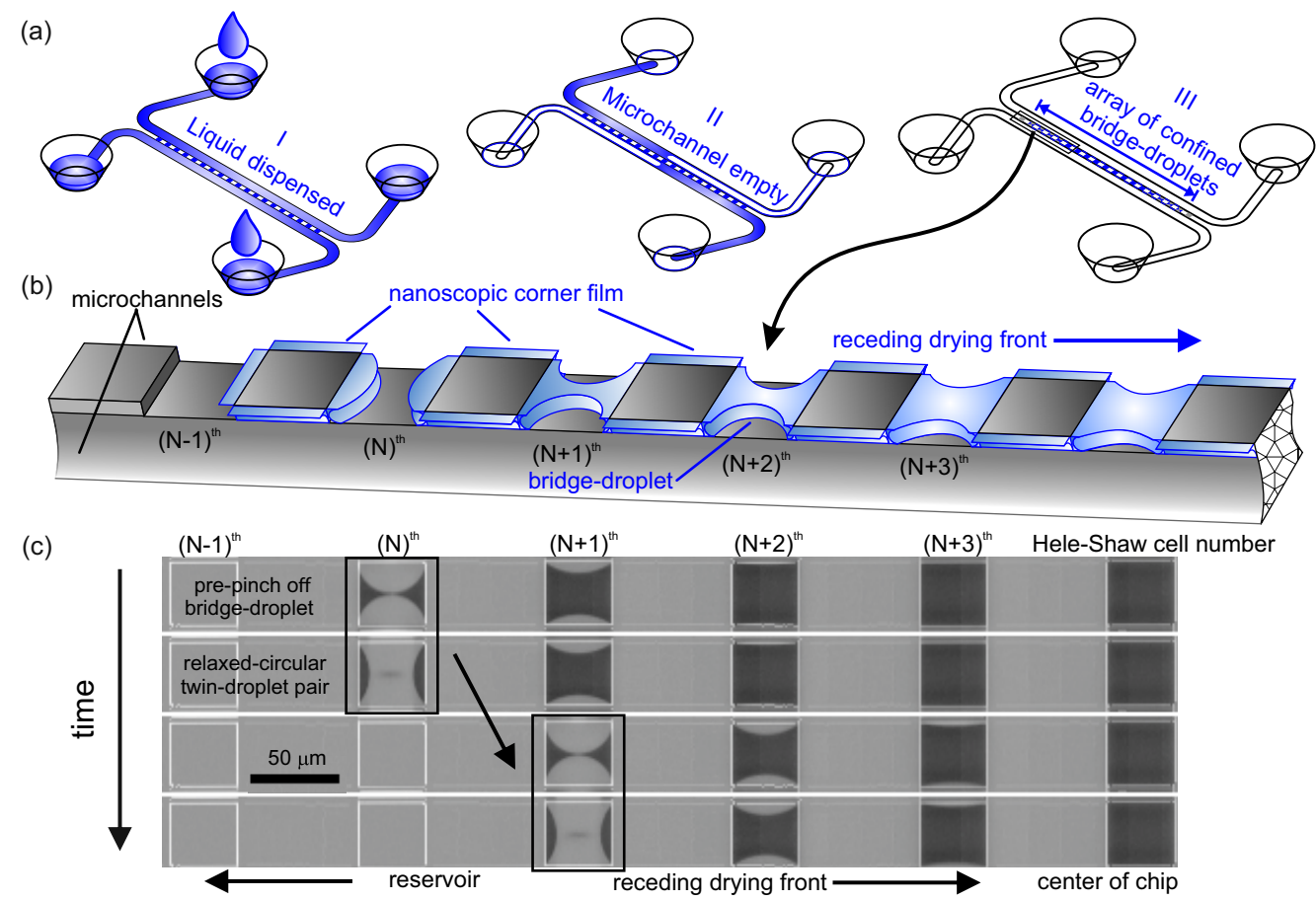

FIG. 2. (a) Liquid is first dispensed into the microchip (I). Evaporation begins at the reservoirs followed by the drainage of microchannels (II) and finally the array of confined bridge-droplets undergoes drainage and evaporation simultaneously (III). (b) Three-dimensional (3D) view of the array of confined capillary bridge droplets (the liquid is blue and the glass microchip structure is light gray). (c) Two consecutive pinch-off events in the nearest neighbor Hele-Shaw cells. Subsequently, the relaxed twin droplets pinned at the edge of Hele-Shaw cell (liquid is dark gray and vapor-filled cells are light gray). 
In nature, the coalescence and breakup of a droplet or bubble forms a spectacular yet simple example of topological change driven by the underlying dynamics [31]. The first pinch-off event occurs in the cell closest to the reservoir due to a higher evaporative demand, after which the pair of nanoscopic corner films do not extend to the microchip reservoir but continue to exist among the nearest neighbor bridge droplets [see Figs. 2(a)III and 2(b)]. Subsequently, the pinch-off continues to occur in each cell (one at a time) proceeding systematically toward the center of the microchip [see Fig. 2(c)]. At this stage, the global mass transport is governed by the vapor diffusion through microchannels. The pinch-off in each Hele-Shaw cell marks the beginning of the observation window. We utilize a high-speed video camera Phantom 2016 v1612 in combination with an optical microscope to capture the droplet shape evolution immediately after the pinch-off. The 12-bit images are acquired at 100000 frames per second (fps) and are subsequently processed via a custom MATLAB script to extract the relevant parameters.

\section{RESULTS AND DISCUSSION}

\section{A. Post-pinch-off dynamics}

Each bridge droplet splits into three droplets, indicating two symmetric pinch points along the neck. The same behavior is observed for five different liquids in hundreds of consecutive cells, as shown in Figs. 2(b) and 2(c) and the Supplemental Material videos [28].

The two symmetric pinch points might originate from an instability along the neck of the bridge droplet due to a small static bending of the walls [32]. The small variation in the gap may originate from the thermal bonding of wafers used in the fabrication process. Alternatively, the two pinch points could result from the underlying dynamics of an unforced Hele-Shaw cell [33]. According to Ref. [33], four pinch-off scenarios are possible for wetting liquid films: The droplet breaks with (1) two pinch points moving toward each other, (2) two asymmetric pinch points propagate away from each other, (3) a single symmetric pinch point, and (4) the droplet relaxes to a stable equilibrium shape without breakup. The number of droplets formed after the pinch-off may vary between two to three, depending upon the number of pinch points [33]. The pinch-off mechanism in the Hele-Shaw geometry is a highly debated subject in theoretical works [33] because no relevant experiment has been reported to date. The exact mechanism of pinch-off is beyond the scope of this article, and understanding it requires a systematic exploration of the gap size scaling and its precise geometry. The pinch-off driven by spontaneous evaporation in Hele-Shaw geometry as reported here can provide a suitable experimental platform to settle this debate.

Across the pinch-off event, there is a measurable change in the internal liquid pressure $\Delta P_{l}$, while the change in the equilibrium vapor pressure $\Delta P_{v}$ is negligible, as the Kelvin corrections corresponding to the change in the in-plane curvature are insignificant. For wetting liquids, the Laplace pressure jump across the liquid-vapor interface is given by $\Delta P_{L}=\sigma\left(1 / r_{\perp}+1 / r_{\|}\right)$, where $r_{\perp}$ and $r_{\|}$are the principal radii of curvature. The radius $r_{\|}$represents the in-plane radius of curvature and the $r_{\perp}$ is fixed by the Hele-Shaw gap size $\left(r_{\perp}=-H / 2\right)$. Consequently, the only variable that determines the change in internal pressure is the in-plane radius of curvature, denoted from now on as $r_{\|}=r$. Figures 3(a)-3(d) show the circles that describe the in-plane radius of curvature $r$, which reflects the droplet shape relaxation. The time $t^{*}=0$ corresponds to the pinch-off. The time axis in Fig. 3(b) is nondimensionalized by using the capillary velocity $v_{C a}$ and half the cell width $W / 2$, as $t=W t^{*} / 2 v_{C a}$. Corresponding to the abrupt topological change, the sign of the in-plane curvature $1 / r$ changes at the dimensionless time $t^{*}=0$ [Fig. 3(b)]. Prior to the pinch-off, the in-plane curvature of the bridge droplet $1 / r_{b}=2 / f W$ is set by the geometry $(f=1.15$; see the Supplemental Material [28] for details). After the pinch-off, the minimum in-plane radius of curvature is positive, $r=r_{i}$, as recorded in the first frame. Thus, the change in internal pressure across the pinch-off is written as $\Delta P_{l}=\sigma\left(1 / r_{i}-1 / r_{b}\right)$. For the experiment with water, the initial curvature is $W / 2 r_{i}=6.39$. This corresponds to the highest recorded point in Fig. 3(f). For water, the estimate of the internal pressure change across the pinch-off is $\Delta P_{l}=37.37 \mathrm{kPa}$. The Kelvin 
(a)

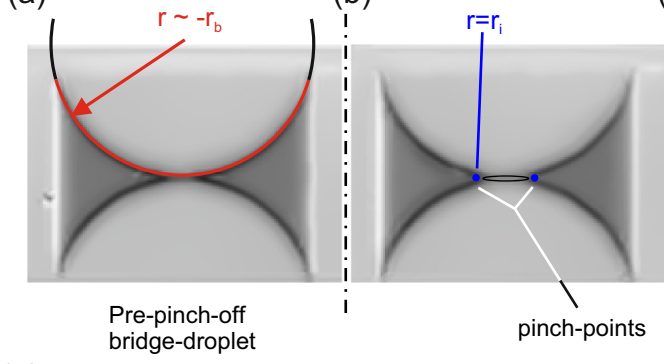

(e)

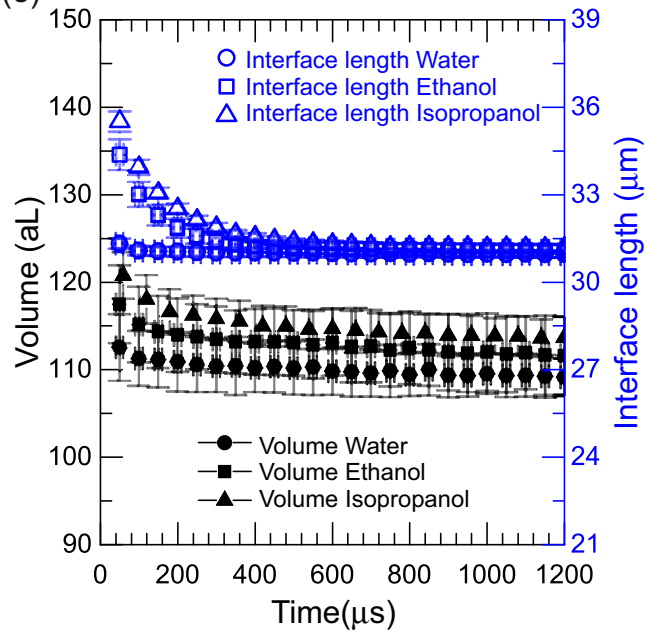

(c)

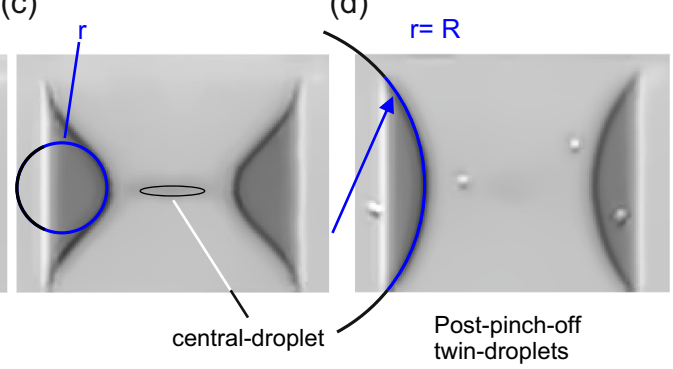

(f)

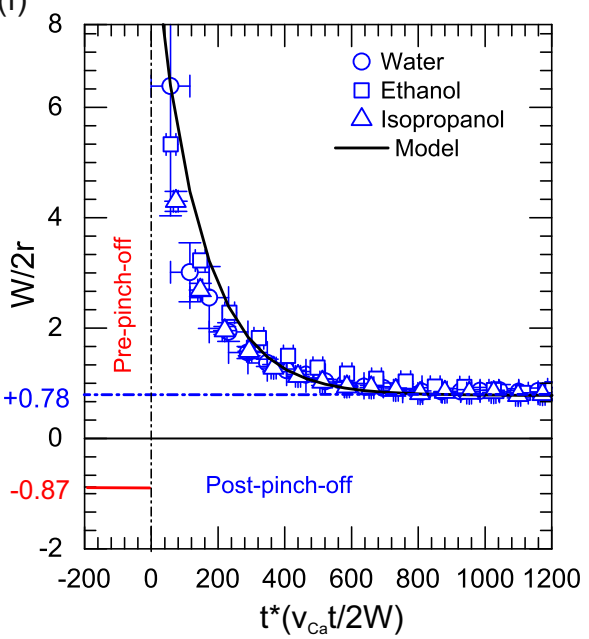

FIG. 3. (a) Bridge droplet before the pinch-off. [(b)-(d)] After pinch-off, the identical pointed droplets relax to a circular shape (increasing time from left to right). Liquid is dark gray. (e) The droplet volume (solid black symbols) and its liquid-vapor interface length (open blue symbols) evolution as a function of time. (f) Plot of $W / 2 r$ (open blue symbols) as a function of dimensionless time $t^{*}$. The droplet in-plane curvature is $1 / r$ and the time axis is nondimensionalized using the ratio of half the Hele-Shaw cell width $W / 2$ and the capillary velocity of the liquid $v_{C a}$.

equation relates the equilibrium vapor pressure $P_{v}$ near a curved liquid-vapor interface and the Laplace pressure jump across it, i.e., $\ln \left(P_{v} / P_{\text {sat }}\right)=\left(\sigma V_{m} / R_{0} T\right)(1 / r-2 / H)$, where $P_{\text {sat }}$ is the saturation vapor pressure for a flat liquid-vapor interface (for water $3.16 \mathrm{kPa}$ ), $V_{m}$ is the molar volume (for water $18 \times 10^{-6} \mathrm{~m}^{3} / \mathrm{mol}$ ), $R_{0}$ is the universal gas constant $(8.314 \mathrm{~J} / \mathrm{mol} \mathrm{K}$ ), and $T$ is the temperature $(298 \mathrm{~K})$ [34]. The shift from the equilibrium vapor pressure across the pinch-off is small, about $\Delta P_{v}=0.28 \mathrm{~Pa}$. The Kelvin corrections are insignificant with regard to the saturation vapor pressure on the order of kilo-Pascals for water.

After the pinch-off, the evaporative effects on the twin droplets are negligible, as shown by the volume versus time plot in Fig. 3(a). First, the equilibrium vapor pressure change $\Delta P_{v}$ is negligible. Second, the central satellite droplet with relatively larger curvature acts as the sacrificial droplet, which feeds any evaporative demand within the cell. Further, during the shape relaxation phase, the liquid from the twin droplets cannot be drained by the nanoscopic corner films as the hydrodynamic resistance to the flow is large. The hydrodynamic resistance $R_{H}$ shows a power-law dependence on the size, for a corner film [35] $R_{H} \propto(H / 2)^{-4}$ and for a Hele-Shaw cell [36] $R_{H} \propto(H)^{-3}$. As a result, the liquid is preferably redistributed within each individual cell, as shown in Figs. 3(b)$3(\mathrm{~d})$. Thus, the volume-preserving and interface-shortening flow demonstrates the two-dimensional nature of the shape relaxation process. 
(a)

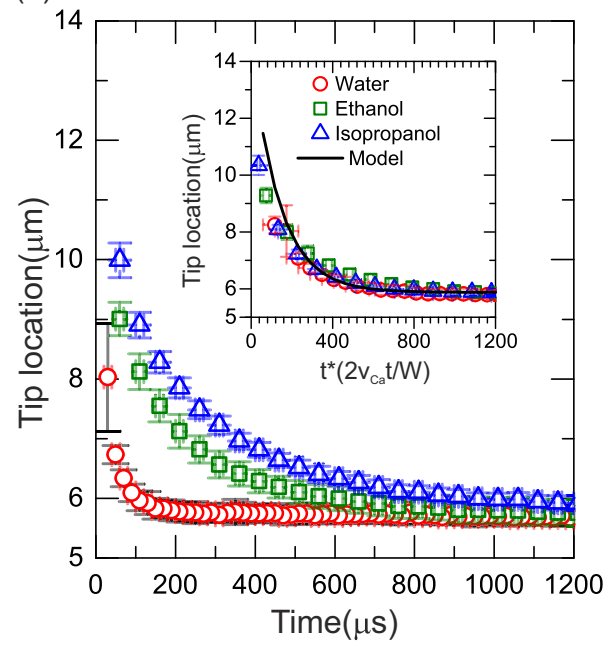

(b)

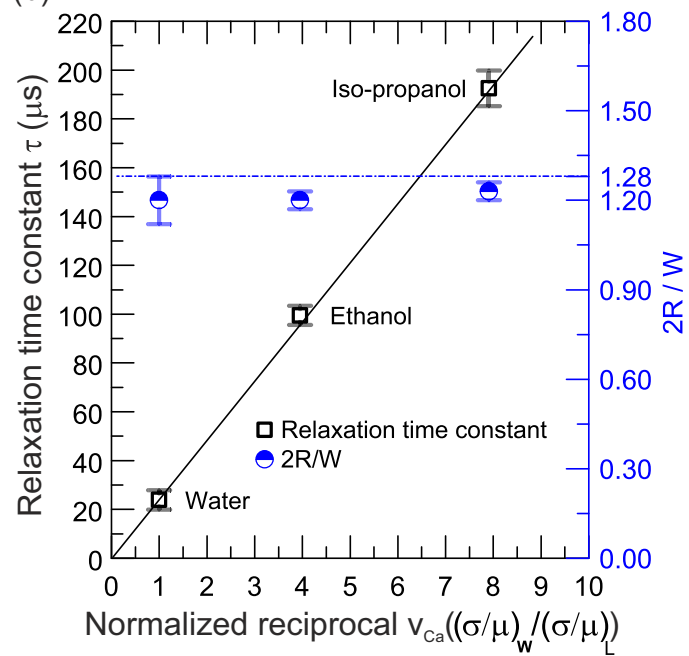

FIG. 4. (a) The tip location evolution as a function of time. The inset highlights that the tip location is concurrent on to a single master curve when plotted as a function of nondimensionalized time $t^{*}$ (using the ratio of half cell width $W / 2$ and capillary velocity of the liquid $v_{C a}$ ). (b) The experimental relaxation time constant $\tau$ (left $y$ axis, black symbols) and the final equilibrium radius of droplets $R /(W / 2)$ (right $y$ axis, solid symbols) vs the normalized reciprocal capillary velocity of liquids with regard to water. The blue line corresponds to the equilibrium radius for the droplets, $R /(W / 2)=1.28$.

After pinch-off, the base of each twin droplet remains pinned at the edge of the lyophilic HeleShaw cell. The pointed shape of the twin droplets is not an equilibrium shape due to the pressure difference between the tip and the base. As a result, the 2D droplets are in the shape relaxation regime. The droplet tip dynamics shows a direct correspondence to the capillary velocity $v_{C a}$. The liquid with higher viscosity or lower surface tension takes a longer time to acquire an equilibrium shape. The tip location and its curvature as a function of dimensionless time $t^{*}$ collapse onto a single master curve for different liquids, indicating that the capillary-viscous regime shapes the 2D relaxation process [see Fig. 4(a) inset and Fig. 3(b)].

\section{B. The relaxation model}

In Hele-Shaw geometry, the depth-averaged Reynolds number compares the ratio of inertial and capillary forces (as the classical Ohnesorge number is irrelevant). The depth-averaged Reynolds number is calculated using the depth-averaged velocity in Hele-Shaw geometry [37]

$$
\bar{u}=-\frac{H^{2}}{G \mu} \nabla P,
$$

where $G=12$ is the constant for an ideal slit geometry and $\nabla P$ is the pressure gradient defined as the pressure drop $\Delta P \sim \sigma / r$ divided by the viscous dissipation length $\approx W / 2$. The expression of the depth-averaged Reynolds number is given by

$$
\overline{\mathrm{R}} \mathrm{e}=\frac{\rho \bar{u}^{2}}{\left(\frac{\sigma}{r}\right)}=\frac{4 \rho \sigma H^{3}}{G^{2} \mu^{2} W^{2}}\left(\frac{H}{r}\right) .
$$

Subsequently, the conservative estimate of $\bar{R} e$ using $r=2.5 \mathrm{H}$ indicates that the inertia does not play any role during the shape relaxation process (see Table I).

The interface evolution based on the images indicates that the tip region can be approximated by the parabolic shape [38]. Shifting the origin of coordinates to the apex of the tip and mirroring the 

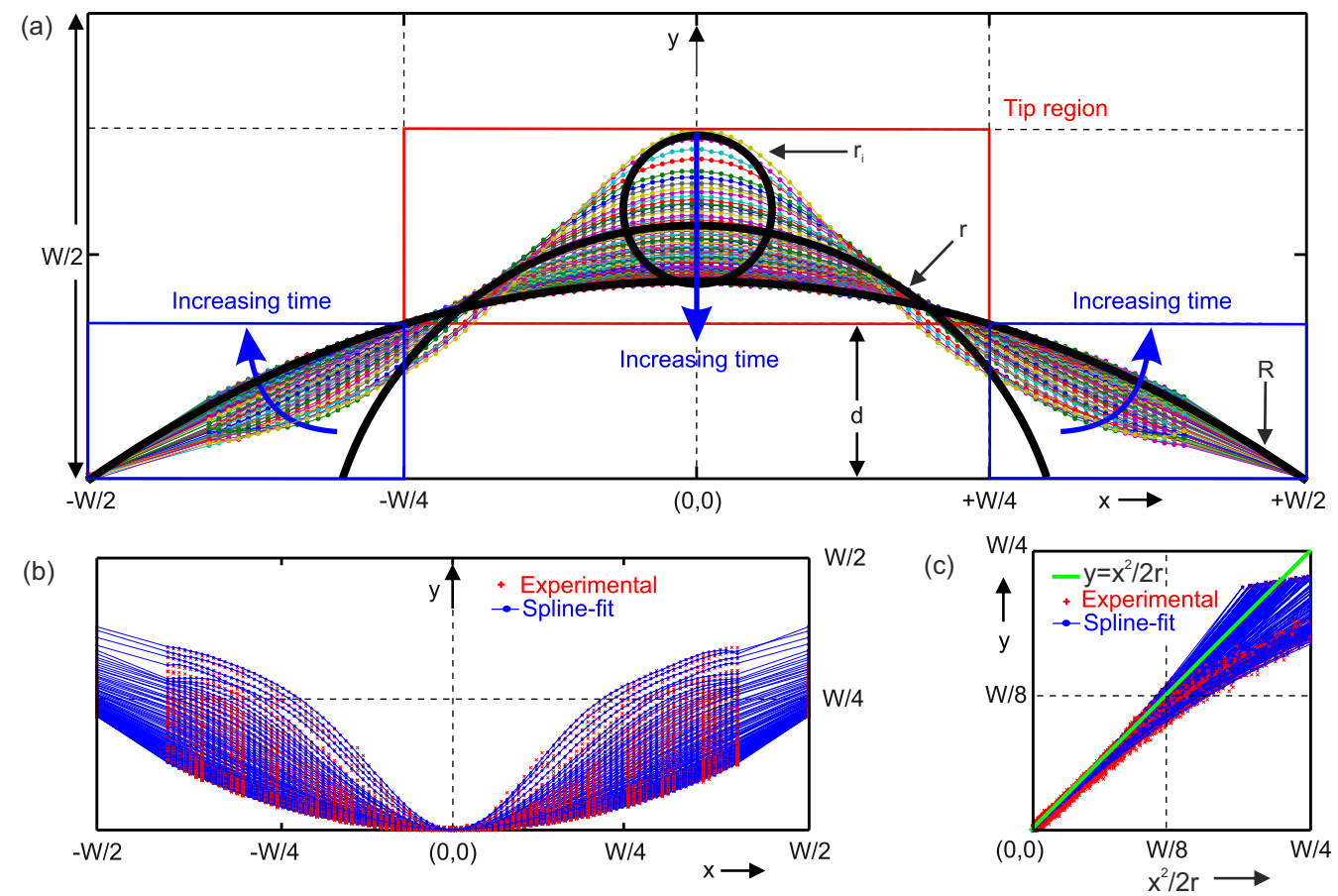

FIG. 5. (a) The temporal evolution of the liquid-vapor interface is marked by different colors with $10-\mu \mathrm{s}$ temporal resolution. The data are marked by solid circle symbols and the lines correspond to the cubic spline-fit curves. The radius of curvature at the tip $r$ is marked by black circles. The tip region lies between $-W / 4 \leqslant$ $x \leqslant+W / 4$ as marked by the red box. (b) Liquid-vapor interface in the transformed coordinate system. The origin is shifted to the tip and the $y$ axis is mirrored. (c) Plot of $y$ vs $x^{2} / r$, where $r$ is the in-plane radius of curvature at the tip. The $(x, y)$ data in panel (c) corresponds to the transformed coordinate system as shown in panel (b). The liquid-vapor interface data as extracted from the images is marked by red solid symbols and the corresponding cubic spline-fit curves by the blue line. The green line indicates $y=x^{2} / r$. Thus, the transformation of liquid-vapor interface data from panels (a) to (c) demonstrates the self-similar parabolic tip evolution.

$y$ axis leads to the result shown in Fig. 5(b). Subsequently, the $x$-axis data are rescaled as $x^{2} / 2 r$ to obtain Fig. 5(c), which validates the parabolic approximation [39] and hence, the tip evolution is self-similar [40]. Figure 5 is based on the data for isopropanol (see Supplemental Material Figs. S3 and S4 for the other liquids [28]). In Fig. 5(a), the equation of the parabola with curvature $1 / r$ that passes through the fixed point at $y=d$ and $x=W / 4$ is given by

$$
y=d-\frac{x^{2}}{2 r}+\frac{W^{2}}{32 r} .
$$

Figure 5(a) shows that the liquid in the tip, within the parabola in the central half of the cell, flows out to the sides, to the two outer quarters of the cell width (see the red and the blue boxes). The volume of liquid in the parabolic tip region, i.e., above $y=d$ and between $-W / 4 \leqslant x \leqslant+W / 4$, is given by $V(r)=W^{3} H / 96 r$. The volume of the parabolic tip region changes as a function of time due to the flux $J=Q / A$, which escapes out at $y=d$ [on the red rectangle in Fig. 5(a)]. The pressure drop $\Delta P=\sigma(1 / r-1 / R)$ occurs over a length scale $\approx W / 2$, where $1 / r$ is the tip curvature and $1 / R$ is the equilibrium curvature of the droplets. Thus, the mean flux using the 
lubrication theory equation (1) is given by

$$
J=\frac{2 \sigma H^{2}}{G \mu W}\left(\frac{1}{r}-\frac{1}{R}\right)
$$

which escapes through the surface area $A \approx H W / 2$ at $y=d$. So, the mass conservation in the parabolic tip region is given by

$$
Q+\frac{d V}{d t}=0
$$

On nondimensionalizing time with the characteristic time constant $\tau=G \mu W^{3} / 96 \sigma H^{2}$, Eq. (5) transforms into $d / d t(1 / r)+1 / r-1 / R=0$ and its solution gives the equation for the evolution of the tip curvature

$$
\frac{1}{r}=\frac{1}{R}+\left(\frac{1}{r_{i}}-\frac{1}{R}\right) e^{-t},
$$

where $r_{i}$ is the initial minimum radius of curvature measured right after the pinch-off. The time $t$ is in units of the relaxation time constant $\tau$. This expression gives a good fit to the experimental data based on $\left(W / 2 r_{i}\right)=6.39, \tau=24 \mu \mathrm{s}$, and $(W / 2 R)=0.78$ [see Fig. 3(b)]. The order of magnitude of $G$ can readily be calculated using the expression for $\tau$, where $W=28 H, H=1 \mu \mathrm{m}$, and $\tau=24 \mu$ s are the typical values for water. As a result, we obtain $G=8.4$, which is close to the theoretical value for an ideal slit. The deviation from the ideal geometrical factor is attributed to the $2 \mathrm{D}$ flow, which is not straight along the axis but diverges towards the droplets' edges [see blue arrows in Fig. 5(a)].

The model shows that the characteristic relaxation time $\tau$ depends on two factors: (I) the capillary velocity of the liquid $v_{C a}$ and (II) the confinement ratio $(C=28)$ of the Hele-Shaw cell. The measured relaxation time constant $\tau$ as a function of the normalized reciprocal capillary velocity [41] shows linearity over one order of magnitude [see Fig. 4(b)]. According to the model, the characteristic relaxation time has a quadratic dependency on the confinement ratio, $\tau \sim C^{2}$. This result is similar to the scaling obtained in Ref. [25], although those results are in the context of droplet merging in Hele-Shaw geometry. Further, the characteristic timescale is also similar to that given in Ref. [42], where the authors numerically study the shapes of viscous liquid blobs (surrounded by an inviscid liquid) subjected to a sink flow in Hele-Shaw geometry. This similarity is attributed to the common approach, i.e., the lubrication approximation.

For a similar liquid film $(\sigma, \mu, H)$, the ratio of the capillary-viscous relaxation timescale between the Taylor-Culick $\left(\tau_{T C}\right)$ and the Hele-Shaw $(\tau)$ experiments scales as $\tau / \tau_{T C} \sim C^{3}$. For $C=28$, the characteristic timescale $\tau$ is about four orders of magnitude longer than the $\tau_{T C}$. This indicates that the departure from the Taylor-Culick mechanism is attributed to the underlaying geometry, which defines the capillary driving pressure and the characteristic viscous dissipation length scale. The observation of the capillary-viscous regime in our experiments is attributed to the geometrical confinement, which substantially slows down the film relaxation dynamics.

\section{Equilibrium radius of droplets $\mathbf{R}$}

The 2D capillary-viscous relaxation results in a droplet with uniform curvature. On considering the volume conservation while neglecting the tiny central droplet, the initial bridge droplet splits into large twin droplets [see Fig. 3(a)]. This gives an estimate of the equilibrium radius of curvature attained by the twin droplets as $R /(W / 2)=1.28$, which agrees with the values measured for the different liquids [see Figs. 3 and 4(b) and the Supplemental Material [28] for more the details]. The circular shape attained by the droplets is a stationary equilibrium state, when compared to the characteristic capillary-viscous timescale. However, on a much longer timescale the twin droplets begin to lose liquid as the evaporative effects and the hydrodynamic conduction through the 
nanoscopic corner films become operational, i.e., about hundreds of milliseconds after the pinch-off. The details of these processes are beyond the scope of the current article.

\section{CONCLUSIONS}

This article presents an experimental study on the shape relaxation of $2 \mathrm{D}$ droplets, formed right after the evaporation-driven spontaneous pinch-off of a capillary bridge droplet, confined in Hele-Shaw geometry. The volume-conserving and interface-shortening flow demonstrates that the relaxation process is essentially two dimensional. After the pinch-off, the tip motion is driven by the capillary pressure due to the in-plane curvature, while the viscous dissipation of the film retards it. As a result, the temporal evolution of the tip shows a self-similar parabolic shape. Based on these observations, the lubrication-approximation model shows that the tip curvature and the evolution of its location follows the exponential decay law. The governing capillary-viscous timescale shows an explicit dependence on the confinement ratio $\left(\tau \sim C^{2}\right)$ and the capillary velocity of the liquid $\left(\tau \sim v_{C a}\right.$ ). Thus, the geometrical confinement ratio facilitates the visualization of the capillary-viscous regime, despite using low-viscosity liquids.

This work demonstrates the capillary-viscous regime for the thin film relaxation, which is complementary to the capillary-inertia regime observed for thick films [2].

The capillary-viscous regime as reported here differs significantly from the Taylor-Culick experiments $[17,18,23,24]$. For a similar liquid film, the ratio of Taylor-Culick and Hele-Shaw film relaxation time constants scales as $\tau / \tau_{T C} \sim C^{3}$. This indicates that the Hele-Shaw confinement leads to a characteristic timescale that is four orders of magnitude longer (for $C=28$ ). This indicates that the departure from the Taylor-Culick mechanism is attributed to the underlaying geometry, which defines the capillary driving pressure and the viscous dissipation length scale.

The current work mainly focuses on the lubrication-approximation-based approach to verify the film relaxation timescale measured experimentally. An extension could be made to numerically solve the Trouton-type model to obtain the 2D flow more accurately and the corresponding droplet shape evolution.

Each bridge droplet splits into three droplets, indicating two pinch points at the neck of the confined bridge droplet. The exact pinch-off mechanism remains an open question, whether its due to the instability originating due to the bending of the walls [32] or a dynamical effect [33]. Understanding the pinch-off mechanism would require a systematic investigation of the Hele-Shaw gap size scaling and its precise geometry. The spontaneous pinch-off driven by evaporation, as observed in the current study, forms an ideal platform to conduct such an exploration. The experimental setup presented here provides an unprecedented control over the volume change, exploiting the coupling between the evaporative effects and the hydrodynamic drainage through the corner films.

The shape relaxation dynamics, as reported here, could be of interest for the laboratory-on-a-chip applications which involve droplet manipulation within the Hele-Shaw geometry.

\section{ACKNOWLEDGMENTS}

D.T. and L.M. designed the experiments. D.T. and J.F.H.-S. performed the experiment. M.D. and D.T. wrote the image analysis protocols and scripts. D.T. and L.M. prepared the analytical model. D.T., J.F.H.-S., H.C., and L.M. cowrote the paper. All authors analyzed the data, discussed the results, and commented on the manuscript. All authors have approved the final version of manuscript.

This work was funded by the French Agency for Research (Agence Nationale de la Recherche), through Grants No. CONGE BLAN-610-01, the Equipex Planex No. ANR-11-EQPX-36, and the Labex Voltaire No. ANR-10-LABX-100-01. D.T. acknowledges support from Dr. Florian Lespinasse (PRISME, Université d'Orléans, France) for loan of the Phantom high-speed camera and Dr. Ida di Carlo (CNRS-ISTO, Orléans, France) for the SEM images. We thank Prof. John Hinch and the anonymous reviewers for the constructive criticism during the review process. 
[1] C. V. Boys, Soap Bubbles, Their Colours, and The Forces Which Mold Them, Vol. 542 (Dover Publications, Inc., Mineola, New York, 1959).

[2] C. Lv, C. Clanet, and D. Quéré, Retraction of large liquid strips, J. Fluid Mech. 778, R6 (2015).

[3] S. J. Weinstein and K. J. Ruschak, Coating flows, Annu. Rev. Fluid Mech. 36, 29 (2004).

[4] E. Villermaux, Fragmentation, Annu. Rev. Fluid Mech. 39, 419 (2007).

[5] E. Villermaux and C. Clanet, Life of a flapping liquid sheet, J. Fluid Mech. 462, 341 (2002).

[6] D. Georgieva, A. Cagna, and D. Langevin, Link between surface elasticity and foam stability, Soft Matter 5, 2063 (2009).

[7] G. Liger-Belair, The physics behind the fizz in champagne and sparkling wines, Eur. Phys. J.: Spec. Top. 201, 1 (2012).

[8] P.-G. De Gennes, F. Brochard-Wyart, and D. Quéré, Capillarity and gravity, in Capillarity and Wetting Phenomena (Springer, Berlin, 2004), pp. 33-67.

[9] C. N. Baroud, F. Gallaire, and R. Dangla, Dynamics of microfluidic droplets, Lab. Chip 10, 2032 (2010).

[10] S. J. Singer and G. L. Nicolson, The fluid mosaic model of the structure of cell membranes, Science 175, 720 (1972).

[11] G. I. Taylor and D. H. Michael, On making holes in a sheet of fluid, J. Fluid Mech. 58, 625 (1973).

[12] F. E. C. Culick, Comments on a ruptured soap film, J. Appl. Phys. 31, 1128 (1960).

[13] W. E. Ranz, Some experiments on the dynamics of liquid films, J. Appl. Phys. 30, 1950 (1959).

[14] L. A. Dupré, Sixième mémoire sur la théorie mécanique de la chaleur, Ann. Chim. Phys. 11, 194 (1867).

[15] L. Champougny, M. Roché, W. Drenckhan, and E. Rio, Life and death of not so "bare" bubbles, Soft Matter 12, 5276 (2016).

[16] C. Cohen, B. D. Texier, E. Reyssat, J. H. Snoeijer, D. Quéré, and C. Clanet, On the shape of giant soap bubbles, Proc. Natl. Acad. Sci. USA 114, 2515 (2017).

[17] G. Debrégeas, P. Martin, and F. Brochard-Wyart, Viscous Bursting of Suspended Films, Phys. Rev. Lett. 75, 3886 (1995).

[18] G. Debrégeas, P.-G. De Gennes, and F. Brochard-Wyart, The life and death of "bare" viscous bubbles, Science 279, 1704 (1998).

[19] L. Rayleigh, Some applications of photography, Nature 44, 249 (1891).

[20] M. P. Brenner and D. Gueyffier, On the bursting of viscous films, Phys. Fluids 11, 737 (1999).

[21] N. Savva and J. W. M. Bush, Viscous sheet retraction, J. Fluid Mech. 626, 211 (2009).

[22] J. N. Israelachvili, Intermolecular and Surface Forces (Academic Press, New York, 2011).

[23] S. V. Chepushtanova and I. L. Kliakhandler, Slow rupture of viscous films between parallel needles, J. Fluid Mech. 573, 297 (2007).

[24] M. Murano and K. Okumura, Bursting dynamics of viscous film without circular symmetry: The effect of confinement, Phys. Rev. Fluids 3, 031601 (2018).

[25] P.-T. Brun, M. Nagel, and F. Gallaire, Generic path for droplet relaxation in microfluidic channels, Phys. Rev. E 88, 043009 (2013).

[26] P. Oswald and G. Poy, Droplet relaxation in Hele-Shaw geometry: Application to the measurement of the nematic-isotropic surface tension, Phys. Rev. E 92, 062512 (2015).

[27] M. Yokota and K. Okumura, Dimensional crossover in the coalescence dynamics of viscous drops confined in between two plates, Proc. Natl. Acad. Sci. USA 108, 6395 (2011).

[28] See Supplemental Material at http://link.aps.org/supplemental/10.1103/PhysRevFluids.3.124202 for the related content. The details of the contents are listed in a readme text file named "Supplemental_content_readme.txt."

[29] P. Concus and R. Finn, On the behavior of a capillary surface in a wedge, Proc. Natl. Acad. Sci. USA 63, 292 (1969).

[30] J. C. T. Eijkel, B. Dan, H. W. Reemeijer, D. C. Hermes, J. G. Bomer, and A. van den Berg, Strongly Accelerated and Humidity-Independent Drying of Nanochannels Induced by Sharp Corners, Phys. Rev. Lett. 95, 256107 (2005).

[31] J. C. Burton and P. Taborek, Role of Dimensionality and Axisymmetry in Fluid Pinch-Off and Coalescence, Phys. Rev. Lett. 98, 224502 (2007). 
[32] L. Keiser, R. Herbaut, J. Bico, and E. Reyssat, Washing wedges: Capillary instability in a gradient of confinement, J. Fluid Mech. 790, 619 (2016).

[33] R. Almgren, A. Bertozzi, and M. P. Brenner, Stable and unstable singularities in the unforced Hele-Shaw cell, Phys. Fluids 8, 1356 (1996).

[34] W. Thomson, 4. On the equilibrium of vapour at a curved surface of liquid, Proc. R. Soc. Edinburgh 7, 63 (1872).

[35] M. Dong and I. Chatzis, The imbibition and flow of a wetting liquid along the corners of a square capillary tube, J. Colloid Interface Sci. 172, 278 (1995).

[36] H. Bruus, Theoretical Microfluidics (Oxford University Press, Oxford, UK, 2008), Vol. 18.

[37] C.-W. Park and G. M. Homsy, Two-phase displacement in hele shaw cells: theory, J. Fluid Mech. 139, 291 (1984).

[38] S. L. Loney, The Elements of Coordinate Geometry (Macmillan, New York, 1920), Vol. 1.

[39] C. V. Raman, Lviii. The curvature method of determining the surface-tension of liquids, London, Edinburgh, Dublin Philos. Magazine J. Sci. 14, 591 (1907).

[40] J. H. Snoeijer, E. Rio, N. Le Grand, and L. Limat, Self-similar flow and contact line geometry at the rear of cornered drops, Phys. Fluids 17, 072101 (2005).

[41] J. A. Dean, Lange's handbook of chemistry, Mater. Manuf. Processes 5, 687 (1990).

[42] E. D. Kelly and E. J. Hinch, Numerical simulations of sink flow in the Hele-Shaw cell with small surface tension, Eur. J. Appl. Math. 8, 533 (1997). 